\title{
Les turbines hydrauliques du barrage des Trois Gorges
}

\author{
The hydraulic turbines of the Three Gorges dam \\ par J. Brémond \\ Ingénieur de Projet - ALSTOM Hydrol \\ G. Vuillerod \\ Dpt Etudes Mécaniques - ALSTOM Hydro
}

As part of the hydroelectric installation of the Three Gorges on the Yangtze river in China, the Alstom group recorded a major order for the supply of 8 Francis turbines out of the 14 to be installed in the left bank power station. This collosal project will include 26 Francis turbines of $710 \mathrm{MW}$, a spillway designed for a maximum flow of $116000 \mathrm{~m}^{3} / \mathrm{s}$, a shiplock of 5 steps with a capacity of 10000 tons and a shiplift of 3000 tons. The concrete gravity dam will be $2.3 \mathrm{~km}$ long.

As oriented by the Specifications, and due to their exceptional size (rumner diameter : $9800 \mathrm{~mm}$ ), the design of these units relies upon well-proofed solutions such as those already experienced on the Itaipu south American large scale hydro project, in which Alstom already contributed 20 years ago. The runners 450 tons each, external diameter $10600 \mathrm{~mm}$ ) will be fabricated by welding of separate elements made of martensitic stainless steel. Most of the components have to be delivered in several parts and reassembled at site by welding or bolting.

The left bank power station is scheduled to be operational in 2006.

\section{I — L'AMÉNAGEMENT HYDRAULIQUE DES TROIS GORGES}

\subsection{Une entreprise gigantesque}

Le programme chinois des Trois Gorges en cours de réalisation sur le fleuve Yang-Tseu représente le plus grand aménagement hydraulique multi-fonction jamais entrepris dans le monde. Le barrage est situé à Sandouping près de la ville de Yichang, dans la province centrale de Hubei. Le projet tire son nom de la succession des défilés sculptés en amont par le fleuve : les majestueuses gorges de Qutang, Wuxia et Xiling.

L'idée de créer une retenue sur le cours du Yang-Tseu est ancienne ; elle est pour la première fois évoquée par SunYatSen en 1919. Après de nombreuses années d'expertises et de discussions, la décision politique de construire le projet des Trois Gorges est finalement entérinée le 3 avril 1992 par un vote de l'Assemblée Nationale Populaire chinoise. Les bénéfices attendus concernent principalement la régulation du cours du Yang-Tseu, la production d'énergie et l'extension de la navigabilité du fleuve.

L'aménagement hydro-électrique actuellement planifié comprend 26 groupes de puissance nominale unitaire $710 \mathrm{MW}$, soit une puissance totale installée supérieure à $18000 \mathrm{MW}$. La première tranche compte 14 unités côté rive gauche de l'ouvrage, dont la conception et la réalisation ont été confiées à des consortiums d'entreprises internationales, avec un important programme de transfert de technologie en direction de l'industrie chinoise. Une deuxième usine en rive droite accueillera 12 autres groupes et il est aussi évoqué une future centrale souterraine optionnelle avec 6 groupes supplémentaires.

\subsection{Huit turbines ALSTOM Hydro}

Le contrat signé le 2 septembre 1997 entre “ Three Gorges International Tendering Company Ltd " (le client), “ China Yangtze Three Gorges Project development Corporation" (le maître d'œuvre), et le consortium Alstom Hydro (France) / Alstom Energia SA (Brésil) prévoit la fourniture des équipements mécaniques de 8 des 14 turbines de la première tranche, et inclut la supervision de montage, la mise en service et le transfert de technologie vers l'entreprise chinoise sous-traitante Harbin Electrical Machinery Co. Ltd, pour un montant total de 212 millions US\$.

Près de 20 années après la participation à la construction du projet Itaïpu à la frontière du Brésil et du Paraguay, ce contrat est pour Alstom Hydro un nouveau grand succès et une référence majeure.

\subsection{L'aménagement en quelques faits et chiffres}

Le barrage est situé au centre du pays à environ $1000 \mathrm{~km}$ des villes de Pékin, Tianjin, Shanghaï et Canton.

1 ALSTOM Hydro est la nouvelle dénomination de la société GEC-ALSTHOM Neyrpic depuis Juin 1998. 


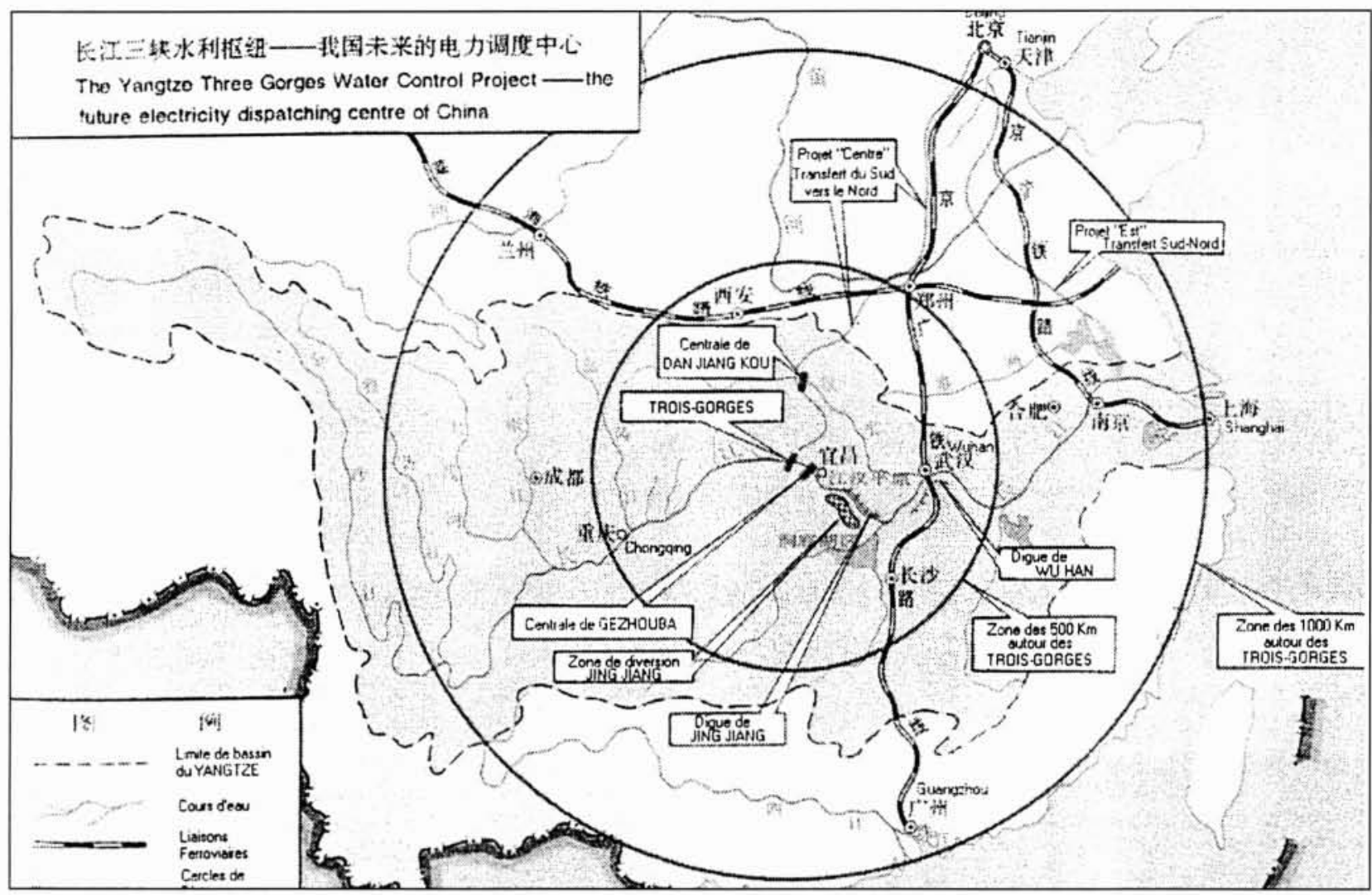

1. Localisation géographique du barrage des TROIS GORGES (d'après document CTGPC [I]).

L'ouvrage retiendra les eaux drainées tout au long des $4500 \mathrm{~km}$ du cours supérieur du Yang-Tseu sur un bassin de plus d'un million de $\mathrm{km}^{2}$ avec un débit annuel moyen de 450 milliards de $\mathrm{m}^{3}$. La prévision de production annuelle d'électricité est de 84,7 milliards de $\mathrm{kWh}$, chiffre qui justifie aux Trois Gorges le qualificatif de plus grand barrage du monde.

Le barrage-poids sera constitué de 15 millions de $\mathrm{m}^{3}$ de béton et aura une longueur de $2345 \mathrm{~m}$.

La capacité totale de la retenue sera de 39,3 milliards de $\mathrm{m}^{3}$ dont 22,15 utilisables en régulation de débit.

Le déversoir est prévu pour un débit maximal de $116000 \mathrm{~m}^{3} / \mathrm{s}$ en crue.

En rive gauche, un canal permettra à des chalands de $10000 \mathrm{t}$ de franchir la dénivellation de $113 \mathrm{~m}$ en cinq degrés d'écluses. Il sera flanqué d'un ascenseur à bateaux de 3000 t de capacité. Un trafic fluvial annuel de 50 millions de tonnes pourra alors remonter le fleuve jusqu'à la ville industrielle de Chongqing à $600 \mathrm{~km}$ en amont du barrage.

Le montant des investissements annoncé par les responsables chinois est pour l'ensemble du projet environ $11 \mathrm{mil}$ liards US\$ dont un tiers doit être consacré au transfert et au relogement des 1,13 millions de personnes déplacées par la mise en eau de la retenue.

\section{D DESCRIPTION DES GROUPES}

\subsection{Caractéristiques générales}

Les principales caractéristiques hydrauliques sont : chute nette nominale : $80,6 \mathrm{~m}$
- chute nette maximale :

$113 \mathrm{~m}$

- puissance nominale :

$710 \mathrm{MW}$

- puissance maximale :

$852 \mathrm{MW}$

- rendement au coeur :

$96,26 \%$

- débit maximal turbinable :

$1065 \mathrm{~m}^{3} / \mathrm{s}$

- vitesse de rotation

$75 \mathrm{tr} / \mathrm{min}$.

Compte tenu du temps nécessaire à la mise en eau progressive du réservoir, les premiers groupes pourront fonctionner sous la chute brute minimale de $61 \mathrm{~m}$ après leur mise en service, ce qui pose une contrainte inhabituelle de conception.

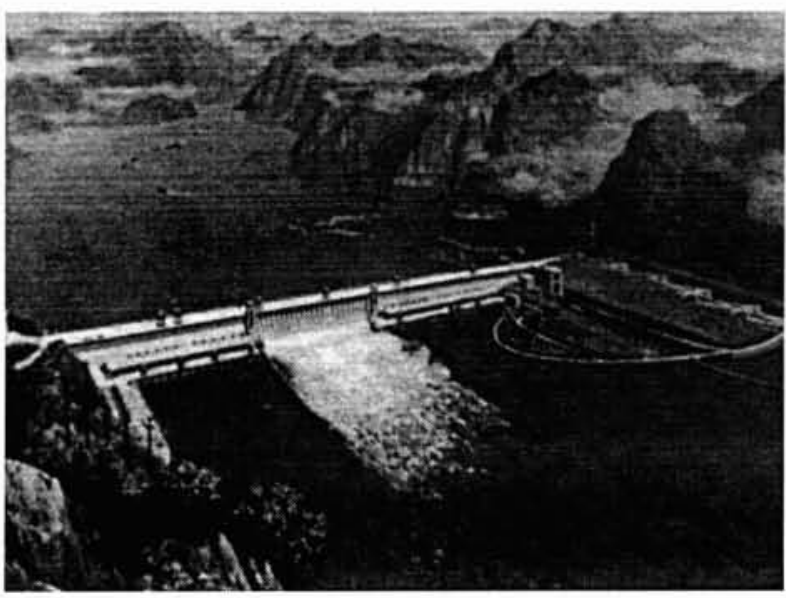

2. Le site futur du barrage

(Source représentation d'artiste CTGPC [1]) 


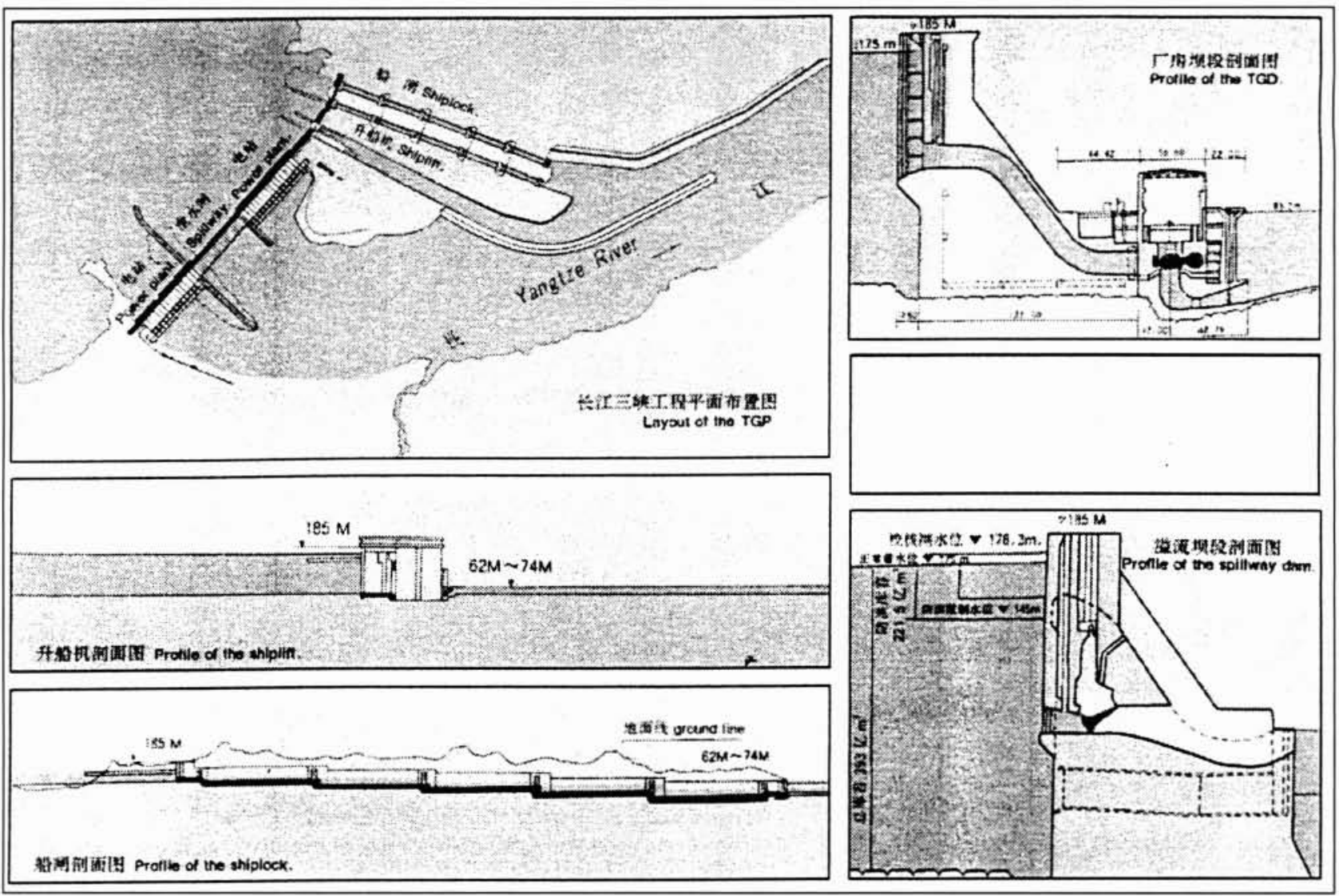

3. Vues schématiques des installations (Source : CTGPC [I])

Les groupes des Trois Gorges sont des turbines de type Francis (vitesse spécifique 262 [tr/min,ch,m]) d'une conception générale relativement classique. En effet, nos interlocuteurs chinois ont toujours souhaité prudemment orienter les constructeurs, notamment par la rédaction des spécifications, vers des solutions techniques déjà éprouvées sur d'autres machines de taille similaire. Il est clair que la référence et l'expérience acquises du fait de notre participation au projet sud-américain Itaïpu nous ont été très utiles pour bâtir et argumenter notre offre.

Cependant, la spécificité remarquable de ces turbomachines réside dans leur taille exceptionnelle puisque le diamètre de sortie de roue est de $9800 \mathrm{~mm}(1,21$ fois la dimension d'ltaïpu), ce qui implique de nouveaux défis pour optimiser le choix et les coûts des matériaux, les joints soudés et les procédés de fabrication, les transports et les manutentions.

Les autres dimensions principales sont les suivantes :

- diamètre du conduit amont à l'entrée de la bâche spirale : $12400 \mathrm{~mm}$

- diamètre de perçage du distributeur :

$11600 \mathrm{~mm}$

- hauteur du distributeur :

$2990 \mathrm{~mm}$

- excentrement de l'entrée de la bâche spirale :

$12500 \mathrm{~mm}$

- distance entre l'axe distributeur et le fond de l'aspirateur : $30000 \mathrm{~mm}$
Passons maintenant en revue les différents composants de cette turbine.

(voir figures 4 : éclaté 3D et 5 : coupe des groupes)

Comme le veut la tradition, nous les décrirons en suivant l'écoulement de l'eau.

\subsection{La bâche spirale}

Elle est constituée d'éléments de volute en acier à haute limite élastique (500 MPa) mécano-soudés en atelier et assemblés puis soudés entre eux sur le site. Elle est conçue

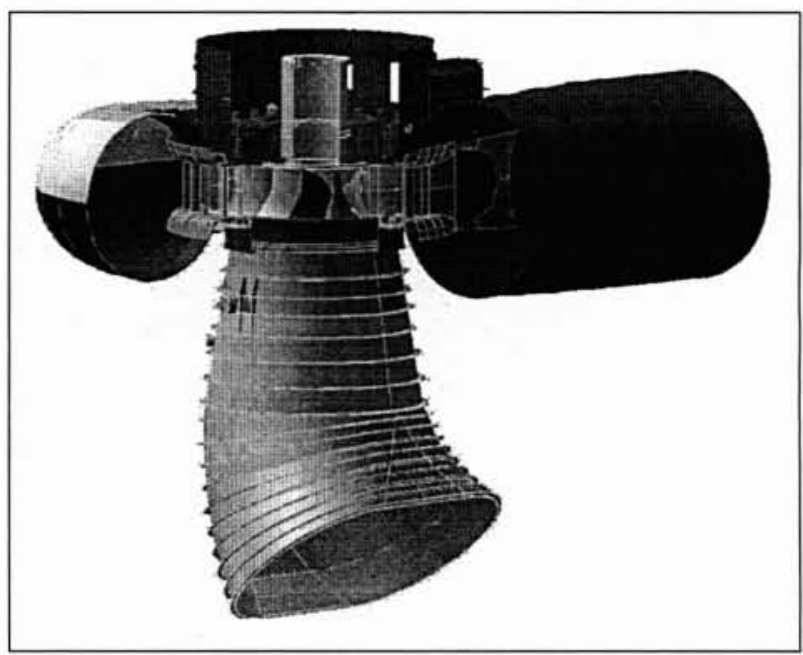

4. Vue éclatée du modèle CAO d'une turbine des Trois Gorges. 


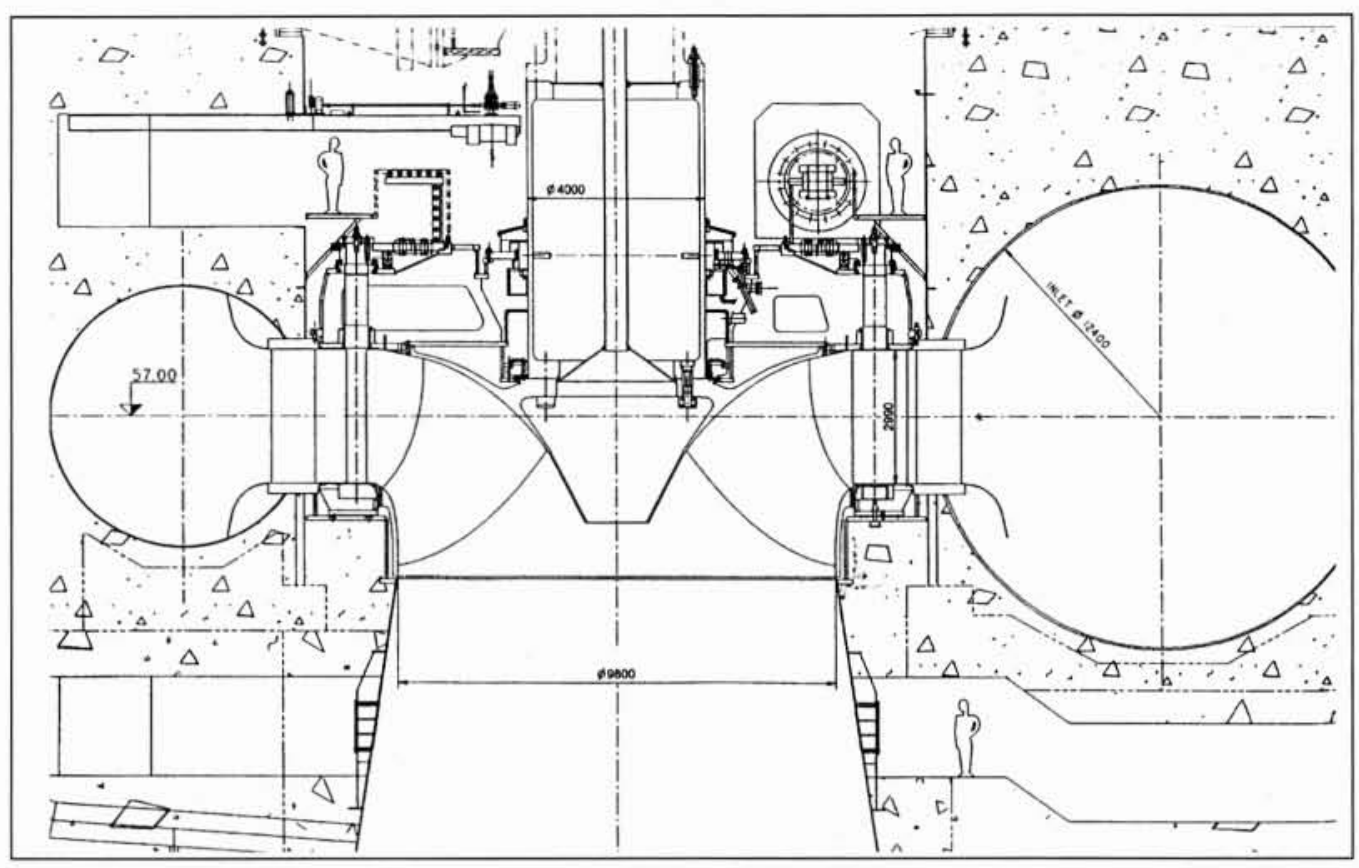

5. Coupe de la turbine.

pour résister à une surpression de 134 m.c.e. Lors du montage et pendant la coulée du béton, elle reposera sur 15 berceaux en béton.

Pendant toute la durée du bétonnage, la pression à l'intérieur de la bâche spirale sera maintenue à une valeur de 70 m.c.e. qui est inférieure à la pression minimale de fonctionnement de façon à assurer en service un appui correct au béton, ce qui contribue à limiter les risques de vibration des groupes.

\subsection{L'avant-distributeur}

Il sera livré sur le site en 6 parties de masse inférieure à 65 tonnes qui ensuite seront soudées entre elles sur site. II est constitué de 2 anneaux en tôle épaisse dont le contour extérieur évolue de l'entrée vers le fond de volute et de 24 avant-directrices. On distingue aussi quatre types différents d'avant-directrices. Des attentes de viroles de la bâche spirale (bavettes) sont soudées sur les anneaux de l'avant-distributeur en atelier, ce qui permet un traitement thermique de détente. Les viroles de la bâche spirale seront soudées sur ces bavettes au chantier.

Pour le maintien de la bâche en pression pendant le bétonnage, un fond d'essai sera soudé sur la manchette amont de la bâche spirale et un faux distributeur fixé à l'intérieur de l'avant-distributeur. Ces outillages pourront être réutilisés sur plusieurs groupes.

\subsection{Le distributeur}

Les flasques supérieur et inférieur, tous les deux de conception mécano-soudée et en quatre parties boulonnées entre elles, supportent 24 directrices orientables en acier inoxydable nuance $\mathrm{Cr}_{13}-\mathrm{Ni}_{4}$, guidées chacune par trois paliers en matériau autolubrifiant, deux sur le flasque supérieur et un sur le flasque inférieur. Chaque directrice a une masse de $9.5 \mathrm{t}$; la partie correspondant au profil hydraulique est cependant une structure creuse en tôles soudées sur les tourillons.

Le flasque inférieur est fixé sur l'anneau de scellement alors que le flasque supérieur est boulonné sur l'anneau supérieur de l'avant-distributeur. Sur chacun des flasques est fixée une plaque d'usure en acier inoxydable qui contient, au droit des directrices en position fermée, un dispositif d'étanchéité en bronze pour réduire les fuites entre directrices et flasques pendant l'arrêt de la turbine. On limite les fuites entre directrices en exerçant un couple de précontrainte par le vannage en fin de course de fermeture.

Les directrices sont orientées au moyen de deux servomoteurs à huile (pression 6,3 $\mathrm{MPa}$ ) par l'intermédiaire d'un cercle de vannage guidé par des secteurs en matériau autolubrifiant fixés sur le flasque supérieur, et d'un ensemble de leviers et de bielles. Un dispositif de rupture en traction sur chaque levier de directrice évite de transmettre des efforts trop importants en cas de coincement d'un corps étranger entre deux directrices adjacentes. Chaque servomoteur est fourni avec un dispositif amortisseur de fin de course de la position de marche à vide à la position complètement fermé.

\subsection{La roue}

La roue est assemblée par soudage à partir d'éléments séparés (moulés et tôles formées) en matériau inoxydable martensitique $\mathrm{Cr}_{13}-\mathrm{Ni}_{4}$. L'expérience d'ALSTOM Hydro et de ses partenaires porte sur un total de 76 roues de diamètre supérieur à $6000 \mathrm{~mm}$ en réalisation mécano-soudée sur les 30 dernières années.(cf. Tableau 1).

Les joints soudés de reconstitution de la ceinture de roue, et d'assemblage des aubes avec le plafond et la ceinture sont prévus en pénétration partielle dans les zones des plus fortes épaisseurs. Outre le fait de réduire les volumes de soudure, cela permet également de mieux maîtriser les déformations en soudage. L'optimisation de la géométrie du joint est validée par des calculs en fatigue, sur des modélisations aux Eléments Finis, et comparés à des résultats de séries d'essais sur éprouvettes. Cette pratique a déjà été appliquée sur plusieurs roues mécano-soudées mises en service par Alstom Hydro.

Les 15 aubes sont entièrement usinées par fraisage sur machine à commande numérique 5 axes ; cette méthode garantit l'excellente similitude des formes géométriques de la 


\begin{tabular}{|c|c|c|c|c|c|c|}
\hline Projet & Pays & $\begin{array}{c}\text { Date de } \\
\text { commande }\end{array}$ & $\begin{array}{l}\text { Nb de } \\
\text { roues }\end{array}$ & $\begin{array}{c}\text { Puissance } \\
\text { (MW) }\end{array}$ & $\begin{array}{l}\text { Chute } \\
\text { (m) }\end{array}$ & $\begin{array}{c}\text { Dia. } \\
\text { Oeuillard }\end{array}$ \\
\hline ITAIPU ${ }^{(*)}$ & $\begin{array}{c}\text { Brésil } \\
\text { Paraguay }\end{array}$ & 1978 & 18 & 740 & 121 & 8100 \\
\hline TUCURUI & Brésil & 1978 & 8 & 360 & 60 & 8150 \\
\hline TUCURUI & Brésil & 1982 & 4 & 360 & 60 & 8150 \\
\hline $\begin{array}{c}\text { AGUA } \\
\text { VERMELHA }\end{array}$ & Brésil & 1974 & 6 & 250 & 57 & 7200 \\
\hline MIRANDA 2 & Portugal & 1991 & 1 & 193 & 55 & 6580 \\
\hline $\begin{array}{l}\text { CABORA } \\
\text { BASSA }^{(*)}\end{array}$ & Mozambique & 1969 & 5 & 415 & 103 & 6560 \\
\hline TEMASCAL & Mexique & 1993 & 3 & 100 & 42 & 5740 \\
\hline EASTMAIN 1 & Canada & 1992 & 3 & 160 & 63 & 5657 \\
\hline LAFORGE 1 & Canada & 1989 & 6 & 142 & 57 & 5580 \\
\hline $\begin{array}{l}\text { TTANSHENG } \\
\text { QIAO }\end{array}$ & Chine & 1995 & 4 & 306 & 111 & 5500 \\
\hline KARRIBA & Zimbabwe & 1995 & 6 & 127.5 & 105 & 4020 \\
\hline KOOTENAY & Canada & 1991 & 4 & 142 & 81 & 5000 \\
\hline KOYNA 4 & Inde & 1994 & 5 & 280 & 500 & 2400 \\
\hline $\begin{array}{l}\text { CHUTEDES } \\
\text { PASSES }\end{array}$ & Canada & 1995 & 5 & 178 & 173 & 3556 \\
\hline WYLIE & USA & 1995 & 3 & 26 & 20 & 4013 \\
\hline
\end{tabular}

(*) en collaboration avec d'autres Constructeurs

Tableau I: Références ALSTOM de roues Francis mécano-soudées

roue construite à celles du modèle réduit essayé et qualifié en laboratoire. Les labyrinthes seront frettés avant usinage final. La roue sera équilibrée statiquement en fin de fabrication.

Compte tenu de ses dimensions (diamètre $10600 \mathrm{~mm}$, hauteur $5080 \mathrm{~mm}$, masse 445 tonnes), sa fabrication, sa manutention et son transport nécessiteront de nombreux outillages spéciaux. L'acheminement du lieu de construction au port d'embarquement de chacune des trois roues assemblées en France constituera sans nul doute un événement sur le parcours, eu égard aux moyens exceptionnels à mettre en œuvre.

\subsection{L'aspirateur}

Le cône est entièrement blindé en tôle d'épaisseur $25 \mathrm{~mm}$, en acier inoxydable sur une hauteur de $1500 \mathrm{~mm}$ en sortie de roue puis en acier noir. Il comporte 2 portes d'accès pour le montage de la plate-forme de visite de la roue.

Le blindage du coude s'étend jusqu'à ce que la vitesse de l'eau atteigne $6 \mathrm{~m} / \mathrm{s}$ environ en son milieu.

La trompette se prolonge jusqu'à 50 mètres à l'aval de l'axe machine. Deux piles, dont le nez est lui aussi blindé, le divisent en 3 conduits ayant chacun une largeur de $9000 \mathrm{~mm}$ et une hauteur de $12400 \mathrm{~mm}$ à la sortie. Un tampon trou d'homme est scellé au plafond du conduit central. Deux tuyaux de vidange obturés par des vannes de diamètre $800 \mathrm{~mm}$ à commande hydraulique sont installés de chaque côté de la trompette.

\subsection{La ligne d'arbre}

L'arbre creux de conception mécano-soudée a un diamètre de $4000 \mathrm{~mm}$. Il ne comporte pas de jupe pour l'appui du palier. Il est relié, en partie basse, avec la roue par des vis ajustées précontraintes, et, en partie haute, avec l'arbre inférieur de l'alternateur par des tiges précontraintes et des douilles creuses.
La ligne d'arbre repose sur 3 paliers et un pivot hydrodynamique. Seul le palier turbine est fourni par Alstom Hydro. Il s'agit d'un palier à 12 patins pivotants lubrifiés à l'huile. La circulation de l'huile est assurée par deux moto-pompes (dont une en stand-by). Des échangeurs eau-huile permettent le refroidissement. Les pompes et les échangeurs sont installés à l'intérieur du flasque supérieur.

\subsection{Le joint d'arbre}

Il est de type axial hydrostatique. Deux anneaux en carbone sont plaqués par l'action combinée de l'eau à étancher et de ressorts sur une plaque d'usure tournante en acier inoxydable. Un débit maximal de $4 \mathrm{l} / \mathrm{s}$ d'eau propre filtrée à 100 microns assure sa lubrification.

\section{CONCLUSION}

Le 8 novembre 1997 les eaux du Yang-Tseu étaient déviées par le canal de dérivation provisoire. Les travaux de l'usine rive gauche pouvaient commencer.

En janvier 1998, soit quatre mois après la signature du contrat, ont été livrées les premières pièces scellées des groupes (blindages des nez de pile). Fin mai suivaient les premiers tronçons d'aspirateur.

La mise en service de la première turbine est programmée en 2003, et les 14 unités de la première tranche doivent être opérationnelles en 2006.

\section{RÉFÉRENCES}

[1] AN EPIC UNDERTAKING - The Three Gorges Project, power for today and tomorrow.- CTGPC, Yichang, Hubei province, China 1994

[2] Conception et construction des turbomachines de grandes dimensions : les turbines Francis de la centrale de Tucurui (Brésil) - Revue technique Neyrpic $n^{\circ} 2-1983$ 